Trauma Surgery \& Acute Care Open

\title{
Alternative payment models: can (should) trauma care be bundled?
}

\author{
Andrew James Kerwin, Alexandra Mercel, David J Skarupa, Joseph J Tepas, Jin H Ra, \\ David Ebler, Albert Hsu, Joseph Shiber, Marie L Crandall
}

Department of Surgery, University of Florida College of Medicine Jacksonville, Jacksonville, Florida, USA

\section{Correspondence to} Dr Marie L Crandall, Division of Acute Care Surgery, Department of Surgery, University of Florida College of MedicineJacksonville, Jacksonville, FL 32209, USA; marie.crandall@ jax.ufl.edu

This study was presented at the 75th Annual Meeting of the American Association for the Surgery of Trauma and Clinical Congress of Acute Care Surgery, September 14-17, 2016, Hilton Waikoloa Village, Hawaii.

Received 5 October 2017 Accepted 17 April 2018

\footnotetext{
To cite: Kerwin AJ, Mercel A, Skarupa DJ, et al. Trauma Surg Acute Care Open 2018:3:e00132.
}

\section{ABSTRACT}

Background Recent legislation repealing the Sustainable Growth Rate mandates gradual replacement of fee for service with alternative payment models (APMs), which will include service bundling. We analyzed the 2 years' experience at our state-designated level I trauma center to determine the feasibility of such an approach for trauma care.

Methods De-identified data from all injured patients treated by the trauma service during 2014 and 2015 were reviewed to determine individual patient injury profiles. Using these injury profiles we created the 'trauma bundle' by concatenating the highest Abbreviated Injury Scale score for each of the six body regions to produce a single 'signature' of injury by region for every patient. These trauma bundles were analyzed by frequency over 2 years and by each year. The impacts of physiology and resource consumption were evaluated by determination of the correlation of the mean and SD of calculated survival probability (Ps) and intensive care unit length of stay (ICU LOS) for each profile group occurring more than 12 times in 2 years.

Results The 5813 patients treated over 2 years produced 858 distinct injury profiles, only $8 \%$ (71) of which occurred more than 12 times in 2 years. Comparison of 2014 and 2015 profiles demonstrated high frequency variation among profiles between the 2 years. Analysis of injury patterns occurring $>12$ times in 2 years demonstrated an inverse correlation between the mean and SD for Ps $\left(R^{2}=0.68\right)$ and a direct correlation for ICU LOS $\left(R^{2}=0.84\right)$.

Discussion These data indicate that the disease of injury is too inconsistent a mix of injury pattern and physiologic response to be predictably bundled for an APM. The inverse correlation of increasing SD with increasing ICU LOS and decreasing Ps suggests an opportunity for measurable process improvement. Level of evidence Economic and value-based evaluations, level IV. Study type Economic/decision.

\section{BACKGROUND}

On March 23, 2010 President Obama signed the Patient Protection and Affordable Care Act (PPACA-PL 111-148), which began the largest overhaul of American healthcare delivery in the history of the country. The basic strategy of the legislation was the extension of coverage to the uninsured, improvement of quality with associated cost containment, and enhancement of population health. PPACA did not address the continued problem of recurring threat to professional reimbursement codified in the Balanced Budget Act of 1997 as the Sustainable Growth Rate (SGR). On April 15, 2015 the SGR was replaced by the Medicare Access and Children's healthcare Reauthorization Act (MACRA-PL 114-10), which reiterated the commitment to link payment with quality, and to replace the traditional fee for service process of reimbursement with alternative payment models (APMs) that include bundled care and shared financial risk. ${ }^{1-5}$ MACRA will begin to overhaul Medicare physician payments beginning in $2019,{ }^{6}$ with the goal of achieving at least $75 \%$ APM by 2025, and as of the end of the first quarter of 2016 the process of transition was ahead of schedule.

The Bundled Payments for Care Improvement (BCPI) was begun in 2013 with the intent of lowering costs and improving quality. ${ }^{7}$ The BCPI is a 3-year experiment designed to test the applicability of the model on 48 different episodes of care. The concept is meant to provide a comprehensive care model redesign which is based on team care to provide the optimum outcome, and coordinated, patient connected care. The key drivers for cost savings would be reductions in the cost of acute care, appropriate utilization of postacute care, and reduction of hospital readmissions. Providers who achieve these cost reductions and quality improvements would be subsequently rewarded for such improvements.

Trauma is a highly variable disease that may not enable cost savings by 'service bundling'. It is not currently included on the list of diseases for the BCPI experiment. ${ }^{7}$ In light of the emerging mandate to assess APMs and in consideration of the growing majority of surgeons who are now employed by hospitals and health systems, we analyzed our level I trauma center's experience to determine if comprehensive care of the injured patient could be bundled. Our hypothesis was that the combination of injuries encountered in the majority of patients would follow a common pattern with predictable severity and need for resources. Processes for bundling trauma care could then be predicated on common injury patterns with expected costs potentially mitigated by severity as defined by the probability of survival. As APM bundling will almost certainly include both parts A and B in Medicare reimbursement, and most trauma surgeons are in an employed status, defining cost of care and determination of reimbursement allocation will be essential to assure physician influence and advocacy in the development of future health delivery systems. 


\section{METHODS}

This was a retrospective review of de-identified data from all injured patients treated by the trauma service during 2014 and 2015. We compiled the highest Abbreviated Injury Scale (AIS) score for each of the six body regions recorded in the trauma registry. Once we had the AIS scores for each body region, we used the process of concatenation to create individual patient injury profiles based on those AIS scores. Concatenation is the process of linking individual items together to produce a chain or series. For this study we concatenated the highest AIS score from each body region together in a series to produce a single number or 'profile'. After concatenation of the AIS scores into the injury profiles, we created the 'trauma bundle' of injury for every patient. These trauma profiles were then analyzed by frequency over the 2-year study period and by each year of the study to determine the variability of incidence of the injury profiles identified. The impact of the various injury profiles on physiology and resource consumption was evaluated by determination of the correlation of the mean and SD of calculated survival probability (Ps) ventilator days (VD) and the intensive care unit length of stay (ICU LOS) for each profile group occurring more than 12 times in 2 years. This low frequency rate of approximately one every other month was intended to capture both high volume and the less common but usually severe injuries that tend to be referred to trauma centers and whose outcome are often influenced by volume experience of the treatment team. The derivation of the three variables is as described below.

\section{Injury profile}

Since its introduction over 50 years ago, the AIS has been considered one of the standards of measurement of injury severity. Because each of the six body regions can be scaled from 0 (no injury) to 6 (immediately fatal), every patient's injury complex can be described as the sequence of AIS proceeding from head through external. Thus, a patient with a subdural hematoma (AIS 3 head), zygoma fracture (AIS 1 face), no thoracic injury (AIS 0 thorax), grade II splenic laceration (AIS 2 abdominal), closed tibia fracture (AIS 2 extremities), and scalp abrasion (AIS 1 external) would have a concatenated profile of 3, 1, 0, 2, 2 and 1. The AIS is most commonly associated with the Injury Severity Score (ISS), introduced by Baker et al in $1974 .{ }^{8}$ The ISS is derived as the sum of the squares of the highest three AIS scores from discrete body regions. Because the ISS reflects the highest AIS score in a specific region, there may be other related injuries that are present and may not be considered by computation of the ISS. For this analysis the AIS profile is intended to serve as a map of injury patterns rather than a comprehensive assessment of mortality risk.

\section{Injury severity}

For the reasons described above the ISS may not reflect all aspects of true mortality risk. For example fatal exsanguination may more likely occur from an AIS 3 liver laceration than an AIS 5 splenic injury. Moreover, the combination of both generates a greater risk than either alone. ${ }^{9}$ Probability of survival (Ps) is calculated using logit probe that includes raising the natural log to power determined by constants that are derived from extensive population analysis of mortality from injury. ${ }^{10}$ Because determination of these constants includes consideration of the ISS and presenting physiology, it serves as a more objective assessment of mortality risk and was used to define ranges of injury severity within specific injury pattern profiles.
ISS Distribution

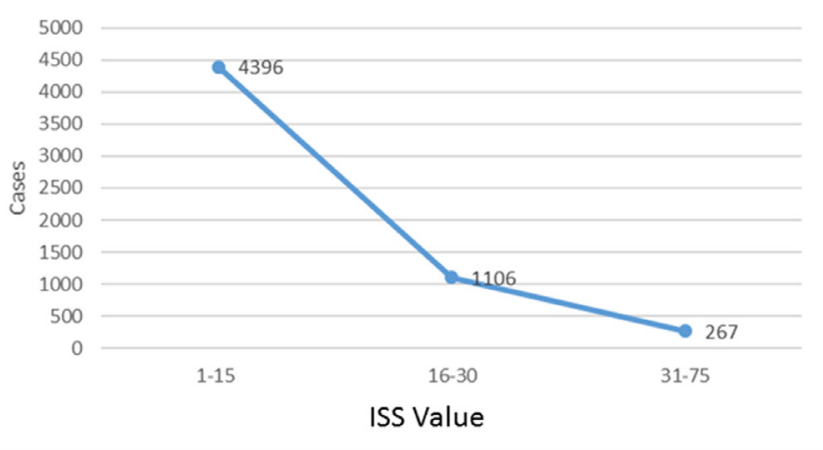

Figure 1 Injury Severity Score (ISS) of the cohort.

\section{Resource consumption}

Determination of cost varies widely among regions and hospital within regions. Since a major determinant of cost of care is ICU $L O S$ and need for mechanical ventilation (VD), these were used in concert with hospital length of stay as surrogates for system cost. Because hospital length of stay can be skewed by additional time awaiting placement, and trauma centers are designed to care for the most severely injured, the primary focus of cost assessment was on injuries requiring intensive care.

\section{RESULTS}

The 5813 patients treated over 2 years produced 858 distinct injury profiles, only $8 \%$ (71 injury profiles) of which occurred more than 12 times in 2 years. These were defined as 'high frequency' (HF) injury profiles because their occurrence was theoretically at a volume that would assure optimal management experience for the trauma service. Of the patients, 4235 (73\%) fell within these $71 \mathrm{HF}$ profiles. In contrast, 92\% (786) of the profiles occurred less than 12 times per year, with some only occurring once. Because 65 patients were treated more than once over the study period, the actual number of encounters for analysis was 6553. Analysis of the study cohort indicates a typical level I trauma center population with $22 \%$ of patients presenting with injury severity greater than ISS 16 (figure 1). Age distribution is demonstrated in figure 2 . The male to female ratio was 67:33. Table 1 demonstrates the 10 most common immediate postresuscitation dispositions. Because the intent of this analysis was determination of feasibility of bundling of trauma care, the injury profiles of all patients, regardless of disposition, were evaluated. The absence of a reliable source of cost encountered in the management of patients who were not admitted required

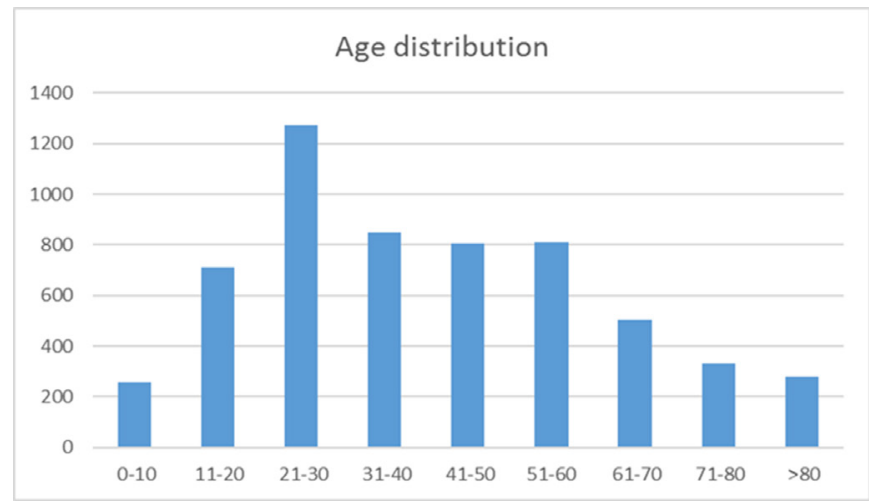

Figure 2 Age distribution of the cohort. 
Table 1 Postresuscitation disposition of the cohort

\begin{tabular}{lll}
\hline Disposition & $\mathbf{n}$ & Percentage \\
\hline Surgical floor & 2090 & 35.9 \\
\hline ICU & 1243 & 21.3 \\
OR & 777 & 13.3 \\
Home & 754 & 12.9 \\
\hline Pediatric ICU & 295 & 5.1 \\
\hline Unknown & 184 & 3.2 \\
\hline Burn center & 96 & 1.6 \\
\hline Dead on arrival & 84 & 1.4 \\
\hline Labor and delivery & 80 & 1.4 \\
\hline Death in trauma bay & 43 & 0.7 \\
\hline
\end{tabular}

$I C U$, intensive care unit; $O R$, operating room.

that the analysis of Ps and resource consumption be focused on inpatients only.

Comparison of calendar years 2014 and 2015 profiles demonstrated a frequency variation between the 2 years. Within the 71 HF profiles $45 \%$ varied by $> \pm 10 \%$ frequency between 2014 and 2015. From the perspective of the feasibility of developing a process of bundling of trauma care, the frequency variation of the HF profiles between study years along with the 786 specific profiles of injury that occurred less that 12 times in 2 years indicates that there is far too much variability among injury patterns to define adequate commonality to anticipate cost of care.

Analysis of the 4235 patients that represent $73 \%$ of the trauma population and fell within the $71 \mathrm{HF}$ injury patterns reinforces this observation. We calculated the mean and SD of the Ps, VD, and ICU LOS for each of the HF profiles that occurred in this study cohort; figures 3-5 provide a visual assessment of the variability among these HF profiles. Figure 3 illustrates the mean and SD of Ps for each profile, the volume of which is listed on the $\mathrm{x}$ axis. The variability among the mean Ps and SD illustrates that within each injury profile, the threat to patient survival and presumed intensity of required care are essentially unpredictable.
For additional clarity, figures 4 and 5, which depict the mean and SD for VD and ICU LOS, respectively, have the respective SDs placed on the negative axis. Again, variability between the mean and SD for both VD and ICU LOS defies predictability of injury profiles regardless of volume of the injury profile.

\section{DISCUSSION}

As the most significant change in healthcare in the history of this country continues to evolve, the impact of MACRA, the legislation that repealed the SGR, is not well understood by most providers. ${ }^{611}$ Like their participating clinicians, many healthcare delivery systems are nowhere near prepared to deal with planned changes in documentation, accountability, and reimbursement. A merit incentive payment system will determine provider incentives or penalties based on a composite score predicated on quality, resource consumption, clinical practice improvement efforts, and advancement of clinical information. The legislation calls for the staged replacement of fee for service by APM with the intent to eventually have less than $25 \%$ of clinical care paid by a fee for service model by 2025 .

What is certain is that the intent of MACRA is optimal quality and increasing cost containment, both of which have for decades been espoused as operating principles of trauma systems. A major component of the MACRA strategy is avoidance of unnecessary expense and use of clinical pathways reflecting best practice that can minimize inappropriate variation in care. ${ }^{12} 13$ This too has been a well-articulated principle of management of trauma care.

From the perspective of trauma system planning, many of these goals have been embedded in the operating principles of trauma care for decades. The questions that must be addressed regarding delivery of trauma care therefore relate to whether there is enough predictability to enable application of some method of injury-based bundling of care. Because accountable care organizations must be capable and willing to assume significant downside revenue risk to be eligible to share in upside savings, it is essential that, in addition to determining whether trauma care can be bundled, the actual costs of this care in whatever system is

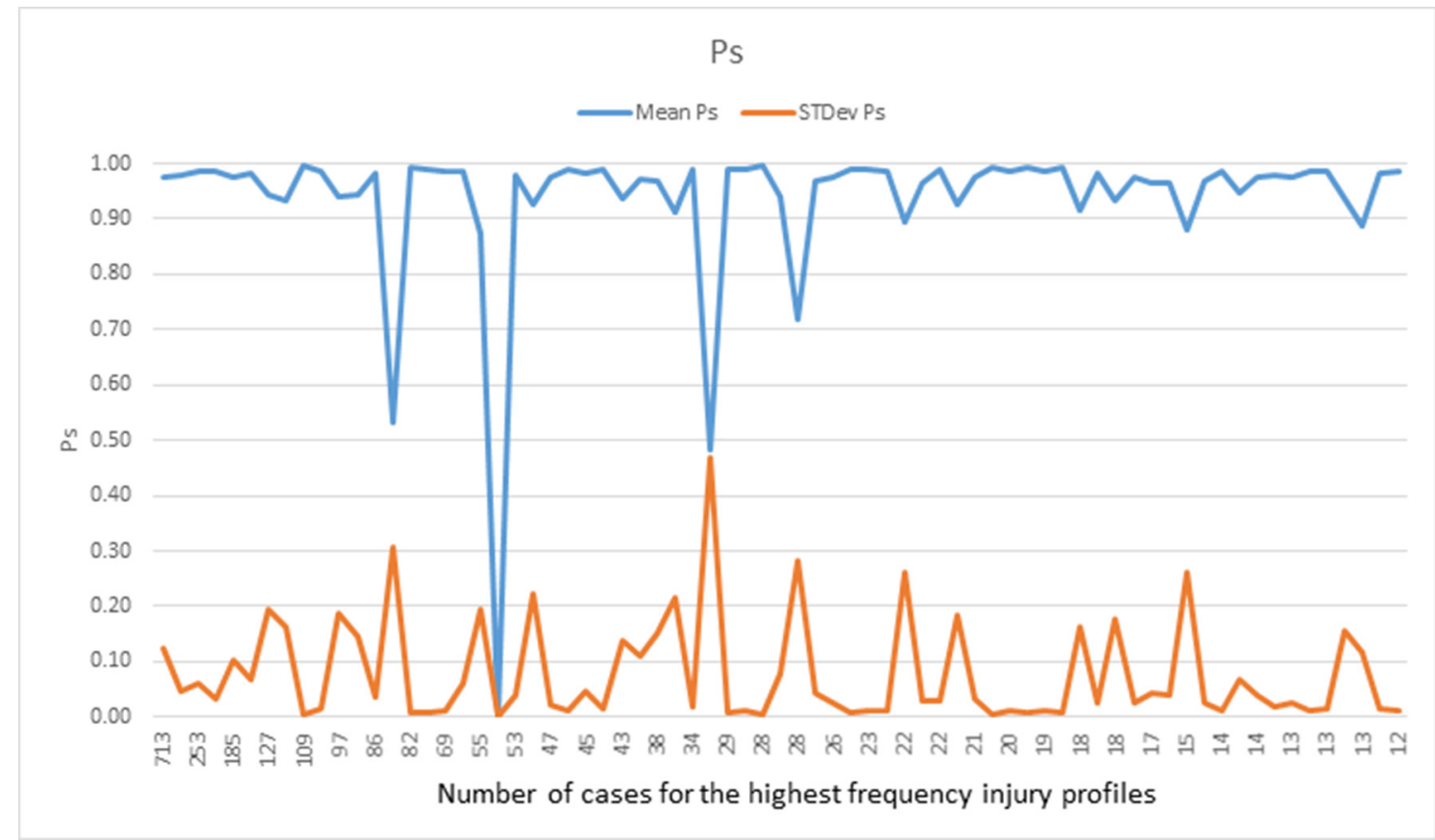

Figure 3 Probability of survival (Ps), mean and SD. 


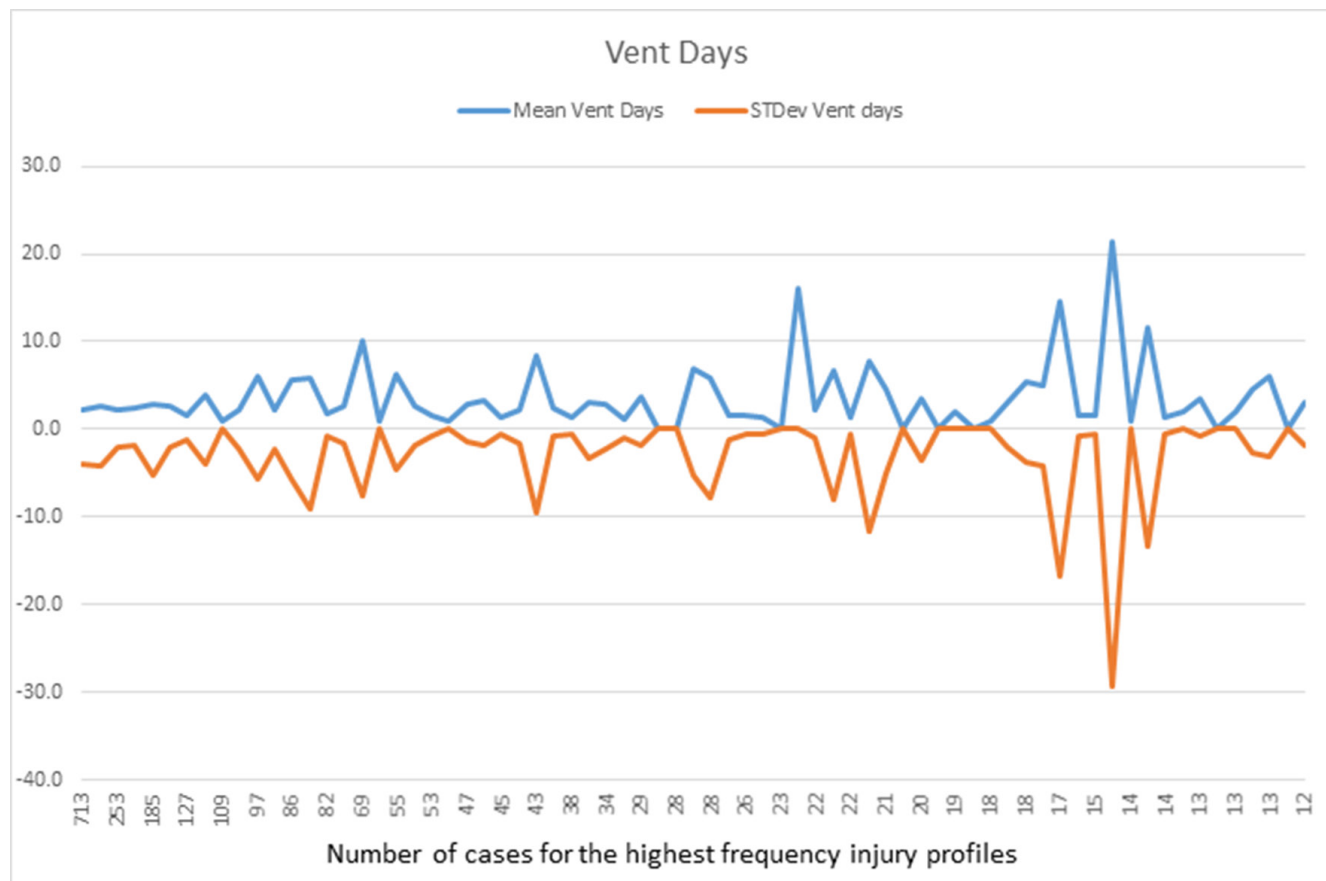

Figure 4 Ventilator (vent) days, mean and SD.

applied must be objectively defined. Moreover, regardless of the payment system that will be required to support trauma care into the future, a process of monitoring cost and its association with severity and outcome must be devised. Accordingly, we sought to assess the feasibility of bundling trauma patient care by specific injury patterns, and, by analyzing the variation of resource use within common injury patterns, to determine whether financial risk within these profiles was predictable.

The AIS system of injury ${ }^{14}$ description has been in use for over 50 years. It was initially devised by engineers to assist in the safe design of highways. The system has been incorporated into the ISS $^{8}$ and into various mortality prediction models devised over the last four decades. Although the AIS has many shortcomings and has been revised multiple times, it does present a description of injured body regions. The convention of the ISS of using just the highest AIS score from the three most severely injured regions limits the accuracy of the ISS in defining the true extent of injury within a body region. Assessment of almost 6000 patients treated over 2 years clearly shows that the variation of regions alone is far too great to define a predictable pattern or

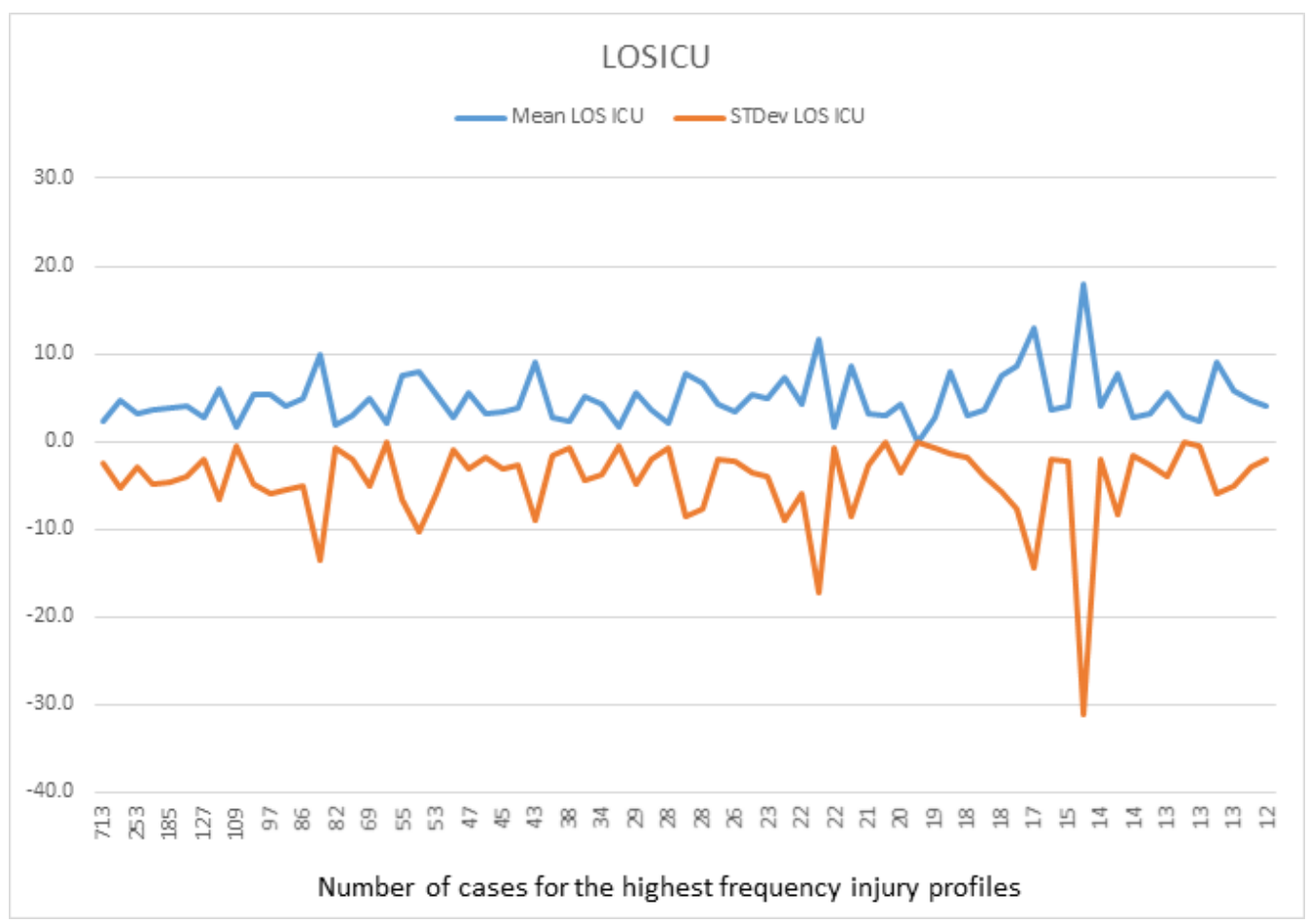

Figure 5 Intensive care unit length of stay (LOSICU), mean and SD. 
patterns of injury that could be expected to define a reliable range of anticipated cost of care. Any attempt to construct a process for predicting cost of care as part of an alternative payment system will obviously need to adjust for many more variables associated with each injured region and every damaged organ. Moreover, the effect of comorbid conditions will also require consideration. Such a predictive model will probably be based on injury inventory rather than a global assessment of cost of care as stratified by the profile of the highest AIS score of injured body regions. From the perspectives of resource consumption and mortality risk, the wide variation within the vast majority of identified patterns further undermines the predictive accuracy of this concatenated body region injury profile, especially when considering unknown and undiagnosed patient comorbid conditions that may also be present.

So, if our model cannot define adequate similarities between our selected patient groupings, is this analysis unlikely to define strategies for preparation for MACRA? From the perspective of defining common profiles of injury, or specific profiles that may be associated with unique needs, monitoring of the association among incidence, severity, and cost of specific injury patterns will inform planners, providers, and payers as to the expense associated with treatment of the disease of injury. This will be especially valuable for population assessment and for global accountability of trauma system function. Theoretically, the dissemination of best practice protocols should be reflected by a narrowing of the variation of cost for high incidence injury patterns. Similarly, determination of drivers of variation of Ps for specific injury patterns will help define best practice. Comparison of these relationship among states or regions may help define more effective prevention and public education strategies.

This study does have some limitations. The major limitation of this analysis is that it represents the experience of a single institution over 2 years. Using only inpatients for that analysis of severity and cost may exclude significant additional findings associated with the process of care of injured patients who do not require admission. It is well known that the per minute cost of care in the resuscitation room of a designated trauma center is among the highest along the continuum of care, so specific findings associated with these types of injuries are not apparent in this data set. This is especially so when considering the impact of 'trauma activation' fees, which have been reported to be as high as $\$ 27000$ at some for-profit institutions. Because this has not been validated elsewhere, interpretation of these results may be limited. Nevertheless, the primary message of significant injury variability and limited predictability of cost clearly indicates that additional research focused on these issues will be essential for effective and equitable implementation of the proposed tenets of MACRA.

Additionally, this study only examines one proposed model for developing an APM for trauma. It does not consider other factors such as the mechanism of injury, age, sex, race, or insurance status. Perhaps some combination of these factors coupled with some, or all, of the highest AIS scores would produce a model that could be used as an APM for trauma care.

\section{CONCLUSION}

The unsustainable cost of healthcare mandates that alternative payment systems provide a manageable and equitable process for reimbursement of comprehensive care of the injured patient.
This includes the entire continuum of support that is required to achieve optimal societal reintegration. Currently the cost of this goal is unknown, and the concept of bundling, which is being considered for so many aspects of healthcare delivery, does not reflect the consistent inconsistency of injury patterns treated so effectively by our nation's trauma systems. Of even greater concern is that absence of objective data regarding predictable costs associated with this care will almost certainly undermine the financial survival of these trauma systems. Trauma surgeons should understand APMs and be actively engaged in the process of trying to develop the appropriate APM for trauma care.

Contributors JJT designed this study. JJT, AM, AJK, and MLC drafted the manuscript. DJS, JHR, DE, JS, and AJK provided editorial assistance. AJK and MLC finalized and submitted the manuscript.

Funding The authors have not declared a specific grant for this research from any funding agency in the public, commercial or not-for-profit sectors.

Competing interests None declared.

Patient consent Not required.

Provenance and peer review Not commissioned; externally peer reviewed.

Data sharing statement Please contact MLC for any data sharing questions. No additional unpublished data are available for this study.

Open Access This is an Open Access article distributed in accordance with the Creative Commons Attribution Non Commercial (CC BY-NC 4.0) license, which permits others to distribute, remix, adapt, build upon this work non-commercially, and license their derivative works on different terms, provided the original work is properly cited and the use is non-commercial. See: http://creativecommons.org/ licenses/by-nc/4.0/

(c) Article author(s) (or their employer(s) unless otherwise stated in the text of the article) 2018. All rights reserved. No commercial use is permitted unless otherwise expressly granted.

\section{REFERENCES}

1 Millard WB. SGR Out, MACRA In. Ann Emerg Med 2016;67:A15-22.

2 Baggot D, Edeburn A. Mandated bundled payments compel hospitals to rethink postacute care. Healthc Financ Manage 2015:69:64-9.

3 Birkmeyer JD, Gust C, Baser O, Dimick JB, Sutherland JM, Skinner JS. Medicare payments for common inpatient procedures: implications for episode-based payment bundling. Health Serv Res 2010;45(6 Pt 1):1783-95.

4 Altman SH. The lessons of Medicare's prospective payment system show that the bundled payment program faces challenges. Health Aff 2012;31:1923-30.

5 Engelman DT. Surgical economics: MACRA, MIPS, and bundles-Lessons learned in the first 3 years of a coronary artery bypass grafting alternative payment model. J Thorac Cardiovasc Surg 2017;153:381-4.

6 Clough JD, McClellan M. Implementing MACRA: Implications for Physicians and for Physician Leadership. JAMA 2016;315:2397-8

7 Greenwald AS, Bassano A, Wiggins S, Froimson MI. Alternative Reimbursement Models: Bundled Payment and Beyond: AOA Critical Issues. J Bone Joint Surg Am 2016;98:e45.

8 Baker SP, O'Neill B, Haddon W, Long WB. The injury severity score: a method for describing patients with multiple injuries and evaluating emergency care. J Trauma 1974;14:187-96.

9 Paddock HN, Tepas JJ, Ramenofsky ML, Vane DW, Discala C. Management of blunt pediatric hepatic and splenic injury: similar process, different outcome. Am Surg 2004;70:1068-72.

10 Boyd CR, Tolson MA, Copes WS. Evaluating trauma care: the TRISS method. Trauma Score and the Injury Severity Score. J Trauma 1987;27:370-8

11 Obama B. United States Health Care Reform: Progress to Date and Next Steps. JAMA 2016:316:525-32.

12 Iorio R. Strategies and tactics for successful implementation of bundled payments: bundled payment for care improvement at a large, urban, academic medical center. J Arthroplasty 2015;30:349-50.

13 Bolz NJ, Iorio R. Bundled Payments: Our Experience at an Academic Medical Center. J Arthroplasty 2016;31:932-5.

14 Anon. Rating the severity of tissue damage. I. The abbreviated scale. JAMA 1971:215:277-80. 